\title{
RAÇA/COR E INDICADORES DE SAÚDE SEXUAL E REPRODUTIVA: IDENTIFICANDO POSSÍVEIS DIFERENÇAS DE COMPORTAMENTO DAS MULHERES NO BRASIL EM $2006^{1}$
}

Angelita Alves de Carvalho ${ }^{2}$

Daniela Polessa Paula ${ }^{3}$

Gabriella de Freitas Vieira ${ }^{4}$

\begin{abstract}
Resumo: Os diferenciais entre brancos e negros são perceptíveis em várias esferas da vida social, tais como a inclusão no sistema formal de educação, mercado de trabalho, até o acesso aos serviços de saúde. Esses diferenciais também estão presentes no acesso à saúde sexual e reprodutiva, com grande desvantagem para o grupo de mulheres negras. Este trabalho tem a finalidade descrever e analisar os diferenciais por raça/cor no acesso à saúde sexual e reprodutiva no Brasil em 2006. Foram utilizados os dados da Pesquisa Nacional de Demografia e Saúde da Criança e da Mulher do ano de 2006. Utilizou-se a análise descritiva, teste Qui-quadrado e modelagem estatística para averiguar associação entre as variáveis. Pode-se concluir que ainda hoje a variável raça/cor influencia o acesso à saúde sexual e reprodutiva. Em alguns indicadores esse efeito é atenuado quando controlado pela escolaridade das mulheres.
\end{abstract}

Palavras-Chave: Saúde da Mulher. Diferenças raciais. PNDS.

\section{RACE AND SEXUAL AND REPRODUCTIVE HEALTH INDICATORS: IDENTIFYING POSSIBLE DIFFERENCES IN WOMEN'S BEHAVIOR IN BRAZIL IN 2006}

\begin{abstract}
The differences between whites and blacks are noticeable in various spheres of social life, such as inclusion in the formal education system, the labor market, even access to health services. These differentials are also present in access to sexual and reproductive health, with great disadvantage for the group of black women. This work aims to describe and analyze the differences by race / color in access to sexual and reproductive health in Brazil in 2006. Data from the National Survey of Demography and Health of Children and Women in 2006 were used. Descriptive analysis, Chi-square test and statistical modeling to verify the association between variables. It can be concluded that even today the race variable influences access to sexual and reproductive health. In some indicators, this effect is attenuated when controlled by women's education.
\end{abstract}

Keywords: Women's Health. Racial differences. PNDS.

\section{Introdução}

As desigualdades sociais no Brasil têm sido amplamente descritas em vários estudos, mas especialmente as desigualdades raciais, étnicas e o racismo têm tido seu debate ampliado e, consequentemente, fomentado políticas públicas com base em diferenciação por raça/cor. Isso se deve tanto pela atuação dos movimentos sociais e também pela constatação, em grande parte dos estudos, de diferenças entre brancos e negros perceptíveis nas várias esferas da vida social, indo desde a inclusão no sistema formal de educação e mercado de trabalho, até o acesso a serviços de

\footnotetext{
1 Versão preliminar foi apresentada no VII Congreso de la Asociación Latino Americana de Población e XX Encontro Nacional de Estudos Populacionais, realizado em Foz do Iguaçu/PR - Brasil, de 17 a 22 de outubro de 2016

${ }^{2}$ Doutora em Demografia pelo CEDEPLAR/UFMG. Atualmente é Pesquisadora e professora do programa de pósgraduação em População, Território e Estatísticas Públicas da Escola Nacional de Ciências Estatísticas (ENCE/IBGE) e do programa de pós-graduação em Economia Doméstica da UFV (litaacarvalho@yahoo.com.br).

${ }^{3}$ Doutora em Engenharia Biomédica pela COPPE/UFRJ, mestrado em Modelagem Computacional pelo Laboratório Nacional de Computação Científica (LNCC). Tem experiência na área de modelagem de dados categóricos, métodos longitudinais e epidemiologia. Atualmente é pesquisadora na Escola Nacional de Ciências Estatísticas (ENCE) (daniela.paula@ibge.gov.br)

${ }^{4}$ Graduada em estatística pela Escola Nacional de Ciências Estatísticas/IBGE (gabrielladefreitasvieira@gmail.com)
} 
saúde (HASENBALG, SILVA, 1998; OLINTO, OLINTO, 2000; MONTEIRO, SAZONE, 2004).

No Brasil, apesar da abolição da escravatura e de muito tempo transcorrido deste então, permanece nos dias atuais a desigualdade e a discriminação racial. Os negros permaneceram à margem da sociedade em vários aspectos sociais e tiveram muitos de seus direitos suprimidos, devido principalmente à ausência de iniciativas políticas para sua inserção social e econômica após a abolição (CAVAS; NETO, 2010; GUEDES, 2014). Não obstante dos negros (grupo formado pela junção dos pretos com os pardos para fins analíticos e por estas duas categorias apresentarem dados sociodemográficos e econômicos similares) representarem mais da metade da população brasileira (quase 55\% do total da população) segundo IBGE (2017), ainda são constatadas inúmeras diferenças quando comparados com o grupo dos brancos no que se refere o direito à cidadania.

Especificamente quanto à saúde e aos diferenciais por raça/cor, pesquisas em diferentes países e regiões têm mostrado desigualdades de morbimortalidade e acesso ao serviço de saúde pela população negra, observado em termos de menor número de consultas e exames de detecção precoce, maiores tempos de espera de exame diagnóstico de patologias, o que implica em menores tempos médios de vida (MADISON et al. 2004; MONTEIRO, 2004; LESSA et al. 2006)

Outros estudos mostram diferenciais importantes no acesso à saúde sexual e reprodutiva bem como aos direitos reprodutivos e sexuais. Corrêa et al. (2006) definem a saúde reprodutiva como o bem-estar físico, mental e social, em todos os aspectos do sistema reprodutivo, seus processos e suas funções, implicando no direito a uma vida sexual segura e satisfatória, e também o direito de homens e mulheres a serem informados e de terem acesso aos métodos eficientes, seguros, aceitáveis e financeiramente compatíveis de planejamento familiar. A saúde sexual é parte da saúde reprodutiva e abrange o desenvolvimento sexual saudável e a ausência de enfermidades, doenças, deficiências, violência e outras práticas de risco relacionadas com a sexualidade. Já os direitos reprodutivos "significam que toda pessoa tem liberdade de escolha para definir como, quando e quantos filhos quer ter[...], além de poder contrair matrimônio de maneira livre e com o pleno consentimento de ambas as pessoas" (CORRÊA et al. 2006,p.48). Os direitos sexuais, por sua vez, buscam garantir a "obtenção do mais alto padrão de saúde sexual, incluindo acesso a cuidados e serviços de saúde sexual e reprodutiva; procurar, receber e concede informação relacionada à sexualidade; educação sexual; respeito pela integridade corporal, escolher seus parceiros; decidir ser ou não sexualmente ativo; ter relações sexuais consensuais; casamento consensual; decidir se, não, ou quando ter filhos; e buscar satisfação, vida sexual saudável e 
prazerosa" (CORRÊA et al. 2006,p.53). Ambos os direitos devem manter “o valor da dignidade inerente ao indivíduo, sendo proibida qualquer discriminação que tenha por base a raça, a etnia, a nacionalidade, a religião, o gênero, a geração, a orientação sexual e demais critérios" (CORRÊA et a. 2006, p. 46).

Contudo, estudos apontam persistentes desvantagens quanto à qualidade da saúde para as mulheres negras, e que estas iniquidades são decorrentes da violação de direitos que dificultam a ascensão social e o acesso a condições dignas dos serviços de saúde por este grupo racial e outras minorias (MONTEIRO; MAIO, 2004; MESENBURG et al. 2018). Por essas e outras razões não é mera coincidência o fato de que a maioria das mortes maternas ocorre entre as mulheres negras, e as principais causas dessas mortes relacionam-se às complicações referentes ao aborto inseguro (LOPES, 2004; MARTINS, 2006; SANTOS et al., 2007). Isso porque as condições em que se dá a maternidade são diferenciadas entre as mulheres negras e brancas, em que as negras apresentam piores condições de assistência ao pré-parto, parto e pós-parto (LEAL et al. 2005)

Perpétuo (2000), que analisou os dados da Pesquisa Nacional de Demografia e Saúde (PNDS) de 1996 e apontou que as negras iniciavam a vida sexual e tinham seu primeiro filho mais cedo que as mulheres brancas, consequentemente, as negras apresentavam maior parturição (61,9\% das mulheres brancas e 48,9\% das mulheres negras tinham de 0 a 2 filhos e 38,1\% das mulheres brancas e 51,1\% das mulheres negras tinham 3 filhos ou mais). A autora também observou que algumas negras, mesmo em idade fértil e sem desejarem engravidar, não faziam uso de métodos contraceptivos, mostrando ainda que esse grupo tinha menor conhecimento sobre a fisiologia reprodutiva. Grande parte das mulheres negras e brancas não havia consultado um médico ao iniciar o uso de pílula anticoncepcional, bem como que negras realizavam menos consultas durante a gravidez e não realizavam consulta após o parto. Diferenciais quanto aos cuidados ginecológicos também foram apontados por Perpétuo (2000) e Simão et al. (2004), os quais relataram que um grande número das mulheres negras nunca fez um exame ginecológico. Esses indicadores mostram diferenciais de comportamento entre os grupos raciais, e revelam em especial, diferenciais no acesso aos serviços básicos saúde sexual e materna. Simão et al. (2004) mostram ainda que as negras realizaram mais consultas ginecológicas no serviço público, enquanto as brancas realizaram mais consultas privadas ou através de convênios.

Como motivos para essas desigualdades, estudos sugerem que a dificuldade de acesso aos serviços de saúde é fruto da discriminação racial, que leva as negras a terem menor escolaridade e renda inferior. Como consequência, possuem menos acesso à planos de saúde e concentram-se mais nas regiões periféricas, onde o sistema de saúde é menos eficaz. Tudo isso gera um quadro 
de acesso precário à saúde sexual e reprodutiva (OLIVEIRA, 1999; PERPÉTUO, 2000; MARTINS, 2006).

Outros estudos (MONTEIRO; SAZONE, 2004; SIMÃO et al., 2004), porém, acreditam que aparentemente, o principal problema não é ser negra mas ser pobre, pois análises mostraram a importância do plano de saúde para que brancas e negras tenham chances iguais de manterem e garantirem uma saúde de boa qualidade. Mais recentemente Berquó et al. (2012) estudaram a reprodução na juventude e encontraram uma grande proporção de jovens negras de menor renda e escolaridade que iniciaram a vida sexual e engravidaram antes dos 20 anos, contudo raça/cor deixou de ser importante para o comportamento sexual e reprodutivo quando as variáveis foram controladas por anos de estudo e classe econômica. Corroborando com essa ideia, Berquó e Lago (2016) mostraram que após análise multivariada, as diferenças entre brancas e negras perderam significância estatística, e outras questões sociais e econômicas se mantiveram como determinantes das iniquidades na atenção em saúde reprodutiva.

Apesar, da perda da significância estatística da variável raça/cor e alguns estudos, não se pode afastar a existência de discriminação racial no acesso a ações de saúde reprodutiva. Como a maioria dos dados só permite avaliar o acesso a ações de saúde, a qualidade do atendimento à saúde disponível para brancas e negras não pode ser mensurada. E, 7portanto, a contemplação do recorte por raça/cor nas pesquisas, especialmente aquelas relacionadas ao acesso à saúde sexual e reprodutiva das mulheres, são fundamentais não só para ampliação do conhecimento acerca do assunto, mas também para a geração de informações que auxiliem no planejamento e implementação de políticas públicas que reduzam as disparidades decorrentes da raça/cor dos indivíduos.

Este trabalho tem como objetivo analisar os diferenciais por raça/cor no acesso à saúde sexual e reprodutiva no Brasil em 2006. Especificamente buscou-se identificar a experiência sexual, contraceptiva e reprodutiva das mulheres segundo raça/cor; compreender os diferenciais por raça/cor com relação ao uso e tipo de contraceptivos, discutindo a demanda insatisfeita por contracepção entre os grupos raciais; analisar o atendimento ao pré-natal por raça/cor e, por fim, conhecer e analisar a implementação das preferências reprodutivas entre brancas e negras.

\section{Metodologia}

Foram utilizados os dados da Pesquisa Nacional de Demografia e Saúde da Criança e da Mulher (PNDS) do ano de 2006. Esta é uma pesquisa amostral com representatividade nacional, que permite inferência para cinco macrorregiões, incluindo o contexto urbano e rural. Foi realizada pelo Ministério da Saúde, coordenada pelo Cebrap e o trabalho de campo ficou a cargo 
do Ibope. A PNDS teve como objetivo contribuir para a melhoria da saúde da criança e da mulher, em que foram investigados temas relativos ao domicílio, reprodução, anticoncepção, conjugalidade, planejamento da fecundidade, entre outros. Seu público alvo envolve mulheres entre 15 a 49 anos totalizando 15575 e informações sobre seus filhos com até 5 anos (PNDS, 2006). Por se tratar de uma pesquisa domiciliar por amostragem probabilística complexa, exigiu a adoção dos procedimentos de inserção do peso, conglomerado e estrato, os quais foram realizados por meio do pacote Complex Sample do SPSS.

Devido ao número pequeno de observações em alguns indicadores para todas as categorias de raça/cor, optou-se se por fazer a agregação do grupo das pretas e pardas em negras. Analisando a literatura sobre estudos raciais verificou-se que essa opção é bastante utilizada, uma vez que, em muitos casos, o comportamento entre os dois grupos é bastante similar (o que também foi verdade no caso deste estudo). Além disso, o percentual de mulheres de outras raça/cor era pequeno, e assim optou-se por analisar somente as diferenças entre negras e brancas. Foram então analisados os casos de 14.625 mulheres brancas e negras de 15 a 49 anos de idade que representam, respectivamente, $40 \%$ e $54 \%$ da amostra total da pesquisa.

Para analisar a saúde sexual e reprodutiva foram utilizados os seguintes temas:

- Experiência sexual e reprodutiva: medida através da idade da primeira relação sexual, idade em que a mulher foi mãe pela primeira vez e o número médio de filhos nascidos vivos.

- Experiência e prevalência contraceptiva: captadas por meio do número de métodos conhecidos, uso de método na primeira relação sexual e atualmente, tipo de método usado na primeira relação e atualmente, e lugar onde a mulher conseguiu o método.

- Planejamento da fecundidade: mensurados por meio da estimação da necessidade insatisfeita por métodos anticoncepcionais e da discrepância de fecundidade. Para o primeiro indicador, utilizou-se o conceito proposto por Westoff e Ochoa (1991), em que a existência da necessidade de métodos anticoncepcionais se daria nos casos em que mulheres que não estão usando métodos e desejam controlar sua fecundidade, seja para adiar o próximo filho desejado ou evitar uma gravidez não desejada depois de ter alcançado o número desejado de filhos ${ }^{5}$.

- Saúde materna: investigou-se o número de consultas de pré-natal ${ }^{6}$ que a mulher realizou durante a gravidez e informações sobre a realização dos procedimentos clínicos básicos ${ }^{7}$. Foram

\footnotetext{
${ }^{5}$ Para maiores detalhes do método ver Westoff e Ochoa (1991) e Lacerda (2005).

${ }^{6}$ Segundo o Ministério da Saúde (2006), o Programa de Humanização no Pré-natal e Nascimento estabelece um mínimo seis consultas de pré-natal, preferencialmente, uma no primeiro trimestre, duas no segundo trimestre e três no último trimestre.

${ }^{7}$ Barros (2013) aponta que os procedimentos clínicos básicos durante a gravidez são: exame de sangue e de urina, medição de pressão e uso de comprimidos contendo ferro.
} 
ainda analisados a variável meses de gravidez quando a mulher realizou a primeira consulta prénatal, local de realização do parto, se houve o acesso a procedimento para alívio da dor durante o parto, se teve acesso à companhia durante o parto e se realizou consulta pós-parto.

Em um primeiro momento realizou-se uma análise bivariada, em que o foco foi a análise dos diferenciais nos indicadores por meio da raça/cor e escolaridade. Para isso, utilizou-se uma estratificação para verificar a influência da escolaridade nos diferenciais inter-raciais, em que a escolaridade foi classificada como baixa e alta, sendo de baixa escolaridade as mulheres com até 8 anos de estudo e de alta escolaridade aquelas com 9 ou mais. Nessa etapa, foram realizados testes Qui-quadrado (considerou-se o nível de significância do p-valor=0,05). Posteriormente fez-se uma análise multivariada em que foram ajustados modelos de regressão linear e logístico fim de se verificar os efeitos das diversas variáveis em conjunto, para além da raça/cor e escolaridade, nos indicadores de saúde reprodutiva. Os modelos bem como suas variáveis estão descritos no quadro abaixo.

QUADRO 1 - Descrição dos modelos de regressão utilizados e variáveis dependentes e explicativas

\begin{tabular}{|c|c|c|c|}
\hline Modelos & Variável dependente & Categorias & Variáveis explicativas \\
\hline \multirow{3}{*}{$\begin{array}{l}\text { Modelo de } \\
\text { regressão } \\
\text { linear }\end{array}$} & idade à primeira relação sexual & contínua & \multirow{3}{*}{$\begin{array}{l}\text { Raça/cor, escolaridade, estrato econômico, } \\
\text { religião, região geográfica, local de } \\
\text { residência, idade da mulher na data da } \\
\text { entrevista }\end{array}$} \\
\hline & Idade ao se tornar mãe & contínua & \\
\hline & número de filhos nascidos vivos & contínua & \\
\hline \multirow{3}{*}{$\begin{array}{l}\text { Modelo de } \\
\text { regressão } \\
\text { logística } \\
\text { binária }\end{array}$} & uso de método contraceptivo & $\begin{array}{c}\text { sim (referência); } \\
\text { não }\end{array}$ & \multirow{2}{*}{$\begin{array}{l}\text { Raça/cor, escolaridade, estrato econômico, } \\
\text { religião, região geográfica, local de } \\
\text { residência, idade da mulher na data da } \\
\text { entrevista, número de filhos nascidos vivos }\end{array}$} \\
\hline & $\begin{array}{l}\text { existência de demanda não } \\
\text { atendida por contracepção }\end{array}$ & $\begin{array}{l}\text { não (referência); } \\
\text { sim }\end{array}$ & \\
\hline & $\begin{array}{c}\text { Número ideal de consultas pré- } \\
\text { natal }\end{array}$ & $\begin{array}{l}\operatorname{sim}(6>= \\
\text { referência }) \\
\text { não }(<=5)\end{array}$ & $\begin{array}{l}\text { Raça/cor, escolaridade, estrato econômico, } \\
\text { religião, região, local de residência, idade da } \\
\text { mulher na data da entrevista, número de } \\
\text { filhos nascidos vivos, possuir plano de saúde } \\
\text { e n }{ }^{\circ} \text { de meses na primeira consulta pré-natal }\end{array}$ \\
\hline \multicolumn{4}{|c|}{ bu } \\
\hline
\end{tabular}

\section{Resultados}

No que envolve o risco reprodutivo entre os grupos raciais (TAB.1) percebe-se que em todos os indicadores este é mais acentuado para as mulheres negras, as quais iniciavam a vida sexual e tinham o primeiro filho mais cedo do que as brancas, muitas delas ainda na adolescência ( $22 \%$ e $30 \%$ das negras contra $14 \%$ e $20 \%$ das brancas, respectivamente). Como consequência, é notória a diferença na parturição das mulheres, e, novamente, entre as de raça/cor negra é bastante elevado o percentual daquelas que tiveram 3 ou mais filhos ( $41 \%$ entre as negras contra $33 \%$ entre as brancas). Estes achados foram estatisticamente significativos, uma vez que no teste Qui-Quadrado obteve-se p-valores inferior à 0,001 para as três variáveis analisadas. 
Ao se comparar com os dados de 1996 descritos em Perpétuo (2000), pode dizer que, houve uma antecipação tanto da experiência sexual quanto reprodutiva entre as décadas. Apesar de se perceber com essa comparação que houve um processo de convergência do comportamento entre os grupos raciais, ainda persistem diferenças, especialmente no que envolve a reprodução em si. Mas de forma geral pode dizer que, nestes indicadores, as diferenças entre os grupos educacionais são mais elevadas do que as diferenças por raça/cor. Entre as mulheres de baixa escolaridade tanto a iniciação sexual quanto o primeiro filho se dão por volta dos 17 anos, enquanto entre as de alta escolaridade estes eventos ocorrem em torno dos 19 e 23 anos, respectivamente. E como resultado, o número médio de filhos é próximo é de em torno à 2,3 para as de baixa escolaridade e 1,4 para as de alta escolaridade.

\begin{tabular}{|c|c|c|c|c|c|c|c|c|}
\hline Indicadores & & Baixa & scolaric & ade & & Alta e & colarid & \\
\hline $\begin{array}{l}\text { Idade que a mulher teve a primeira } \\
\text { relação sexual }\end{array}$ & Branca & Negra & Total & $\begin{array}{l}\text { Teste Qui }^{2} \text { e } \\
\text { de média }\end{array}$ & Branca & Negra & Total & $\begin{array}{l}\text { Teste Qui }{ }^{2} \mathrm{e} \\
\text { de média }\end{array}$ \\
\hline Menor que 15 anos & 14,0 & 22,0 & 19,0 & \multirow{3}{*}{0,000} & 4,0 & 7,2 & 5,7 & \multirow{3}{*}{0,000} \\
\hline De 15 a 17 anos & 42,1 & 43,1 & 42,7 & & 36,9 & 36,2 & 36,5 & \\
\hline 18 anos ou mais & 43,9 & 34,9 & 38,2 & & 59,2 & 56,6 & 57,8 & \\
\hline Idade média à $1^{\text {a }}$ relação sexual & 17,7 & 17,2 & 17,5 & 0,375 & 18,9 & 19,0 & 18,9 & 0,114 \\
\hline $\begin{array}{c}\text { Idade que a mulher foi mãe pela } \\
\text { primeira vez }\end{array}$ & Branca & Negra & Total & Teste Qui ${ }^{2}$ & Branca & Negra & Total & Teste Qui ${ }^{2}$ \\
\hline Até 16 anos & 20,1 & 29,6 & 26,0 & \multirow{4}{*}{0,000} & 6,1 & 12,8 & 9,6 & \multirow{4}{*}{0,000} \\
\hline De 17 a 19 anos & 35,6 & 35,2 & 35,4 & & 21,0 & 30,8 & 26,2 & \\
\hline De 20 a 24 anos & 31,9 & 24,1 & 27,0 & & 36,3 & 31,6 & 33,8 & \\
\hline 25 anos ou mais & 12,4 & 11,1 & 11,6 & & 36,5 & 24,8 & 30,3 & \\
\hline Idade média à maternidade & 17,71 & 17,19 & 17,5 & 0,085 & 24,1 & 22,3 & 23,2 & 0,021 \\
\hline Número de filhos nascidos vivos & Branca & Negra & Total & Teste Qui ${ }^{2}$ & Branca & Negra & Total & Teste Qui ${ }^{2}$ \\
\hline 0 & 9,6 & 9,5 & 9,5 & \multirow{3}{*}{0,000} & 23,5 & 20,7 & 22,0 & \multirow{3}{*}{0,000} \\
\hline De 1 a 2 filhos & 57,2 & 49,8 & 52,6 & & 66,7 & 62,7 & 64,6 & \\
\hline 3 filhos ou mais & 33,2 & 40,7 & 37,9 & & 9,9 & 16,6 & 13,4 & \\
\hline Número médio de filhos & 2,14 & 2,47 & 2,3 & 0,000 & 1,32 & 1,47 & 1,4 & 0,000 \\
\hline
\end{tabular}

Na tabela 2 tem-se os modelos de regressão linear para as variáveis de experiência sexual e reprodutiva apresentadas acima. Percebe-se que somente para modelo de idade à primeira relação sexual a variável raça/cor não foi estatisticamente significativa, ou seja, parece que a raça/cor não tinha efeito para a determinação na iniciação sexual no ano de 2006 , resultado este que difere de alguns estudos regionais tais como o De Souza et al. (2018). Nesse caso, morar na zona rural, em qualquer região, exceto Sudeste, serem de gerações mais jovens e de baixa escolaridade foram os fatores que influenciam na antecipação da iniciação sexual, revelando uma questão geracional e de desigualdade social/cultural/regional interessante sobre a sexualidade das brasileiras. Estes dados corroboram com outros estudos nos quais também foram encontrados determinantes semelhantes para iniciação sexual de jovens (AERTS et al, 2014). Para os outros dois indicadores, reforça-se os resultados já encontrados na análise descritiva. Ou seja, mesmo 
controlando-se por variáveis que, de certa forma, captam o efeito das desigualdades sociais e econômicas entre os grupos raciais, a raça/cor ainda é determinante para a experiência reprodutiva das mulheres. Pertencer a raça/cor negra antecipa o evento da maternidade e aumenta o número médio de filhos, o que também foi encontrado no estudo de Coutinho e Gougher (2018). Além disso, outras variáveis se mostraram relevantes, no caso da idade ao ter o $1^{\text {o }}$ filho, ter baixa escolaridade, pertencer a estratos econômicos inferiores, residir no Sudeste e ser de geração mais jovem antecipam o evento da maternidade. Por outro lado, o aumento da idade, residir no Norte, Nordeste e Centro-Oeste, ter baixa escolaridade e pertencer à baixos estratos econômicos aumentam a quantidade média de filhos nascidos vivos.

\begin{tabular}{|c|c|c|c|c|c|c|c|}
\hline \multirow{2}{*}{$\begin{array}{c}\text { Variáveis } \\
\text { independentes }\end{array}$} & \multirow[t]{2}{*}{ Categorias } & \multicolumn{2}{|c|}{$\begin{array}{c}\text { Idade } 1^{\text {a }} \text { Relação } \\
\mathrm{N}=8492\end{array}$} & \multicolumn{2}{|c|}{$\begin{array}{c}\text { Idade } 1^{\circ} \text { filho } \\
\mathrm{N}=7652\end{array}$} & \multicolumn{2}{|c|}{$\begin{array}{c}\text { Número de filhos } \\
\mathrm{N}=8492\end{array}$} \\
\hline & & Efeito & P-valor & Efeito & P-valor & Efeito & P-valor \\
\hline Raça/cor & $\begin{array}{l}\text { branca } \\
\text { negras }\end{array}$ & $\begin{array}{c}\text { Referência } \\
-0,257\end{array}$ & 0,270 & $\begin{array}{c}\text { Referência } \\
-0,308\end{array}$ & 0,029 & $\begin{array}{c}\text { Referência } \\
0,193\end{array}$ & 0,000 \\
\hline Escolaridade & $\begin{array}{c}\text { Alta } \\
\text { Baixa }\end{array}$ & $\begin{array}{c}\text { Referência } \\
-1,632 \\
\end{array}$ & 0,000 & $\begin{array}{c}\text { Referência } \\
-2,525\end{array}$ & 0,000 & $\begin{array}{c}\text { Referência } \\
0,637\end{array}$ & 0,000 \\
\hline $\begin{array}{c}\text { Estratos } \\
\text { Econômicos }\end{array}$ & $\begin{array}{c}\text { Estratos A e B } \\
\text { Estrado C } \\
\text { Estrado De E }\end{array}$ & $\begin{array}{c}\text { Referência } \\
-1,632 \\
-1,023\end{array}$ & 0,142 & $\begin{array}{c}\text { Referência } \\
-1,484 \\
-2,129\end{array}$ & 0,000 & $\begin{array}{c}\text { Referência } \\
0,121 \\
0,440\end{array}$ & 0,000 \\
\hline Religião & $\begin{array}{c}\text { Católica } \\
\text { Evangélica } \\
\text { Outras e sem } \\
\text { religião }\end{array}$ & $\begin{array}{c}\text { Referência } \\
0,182 \\
-0,643\end{array}$ & 0,049 & $\begin{array}{c}\text { Referência } \\
0,049 \\
-0,412\end{array}$ & 0,038 & $\begin{array}{c}\text { Referência } \\
0,107 \\
-0,083\end{array}$ & 0,030 \\
\hline $\begin{array}{l}\text { Macro região } \\
\text { administrativa }\end{array}$ & $\begin{array}{c}\text { Sudeste } \\
\text { Norte } \\
\text { Nordeste } \\
\text { Sul } \\
\text { Centro-Oeste }\end{array}$ & $\begin{array}{c}\text { Referência } \\
-1,074 \\
-0,032 \\
-0,417 \\
-0,139\end{array}$ & 0,000 & $\begin{array}{c}\text { Referência } \\
-1,306 \\
-0,262 \\
-0,860 \\
-0,515\end{array}$ & 0,000 & $\begin{array}{c}\text { Referência } \\
0,628 \\
0,245 \\
0,187 \\
-0,032 \\
\end{array}$ & 0,000 \\
\hline $\begin{array}{l}\text { Local de } \\
\text { residência }\end{array}$ & $\begin{array}{c}\text { Urbano } \\
\text { Rural }\end{array}$ & $\begin{array}{c}\text { Referência } \\
0,405\end{array}$ & 0,037 & $\begin{array}{c}\text { Referência } \\
0,211\end{array}$ & 0,679 & $\begin{array}{c}\text { Referência } \\
0,093\end{array}$ & 0,114 \\
\hline $\begin{array}{l}\text { Idade da } \\
\text { mulher }\end{array}$ & contínua & 0,454 &, 000 & 0,192 &, 105 & 0,076 &, 000 \\
\hline
\end{tabular}

Fonte: PNDS, 2006

Com relação à experiência e prevalência contraceptiva, a Tabela 3 revela que o desconhecimento acerca do período fértil é geral para as mulheres de baixa escolaridade e ainda pior para as mulheres negras. Quando se observa os dados de Perpétuo (2000) para 1996, podese dizer que houve pouca melhoria acerca deste tipo de informação (em 1996 apenas 20\% das negras e $37 \%$ das brancas responderam corretamente, em 2006, o percentual de negras que declararam a resposta correta foi de $30 \%$ contra $46 \%$ das brancas). No que diz respeito ao número de métodos contraceptivos que conhece, os dados mostram uma proximidade entre os grupos raciais, com uma pequena diferença, não estatisticamente significativa, entre os grupos educacionais. Contudo, essa proximidade do conhecimento sobre os métodos não se mostrou no uso, pois as negras apresentam-se em maior percentual do que as brancas entre aquelas que não 
utilizaram métodos na primeira relação sexual, tanto entre as de baixa quanto de alta escolaridade. Também as negras ficaram em desvantagens quando comparadas às brancas quanto à orientação médica ao utilizarem a pílula, em que um percentual mais elevado delas não consultou médico. Resultados similares foram encontrados em Santos (2015), em que as mulheres negras apresentaram piores condições do que as brancas de acesso à informação e métodos contraceptivos em Recife.

Seguindo esta tendência, as mulheres negras também eram aquelas que mais não utilizavam nenhum método ou eram esterilizadas (TAB.3). Estes diferenciais acabam por resultar em um planejamento diferenciado da fecundidade, pois existia um percentual superior entre as negras com demanda por contracepção, especialmente para limitar o número de filhos. Esse dado somente foi estatisticamente significativo para o grupo mais escolarizado, entre as quais as negras tinham mais chance de não utilizarem nenhum método apesar de serem fecundas e não desejarem mais ficar grávidas. Os diferenciais de raça/cor no acesso e amplitude do mix contraceptivo reduziram em relação aos encontrado por Perpétuo (2000), contudo ainda permanece a falta de informação e menor acesso das negras à saúde sexual.

\begin{tabular}{|c|c|c|c|c|c|c|c|c|}
\hline Indicadores & \multicolumn{4}{|c|}{ Baixa escolaridade } & \multicolumn{4}{|c|}{ Alta escolaridade } \\
\hline Período fértil da mulher & Branca & Negra & Total & Teste Qui ${ }^{2}$ & Branca & Negra & Total & Teste Qui ${ }^{2}$ \\
\hline No meio do ciclo & 31,6 & 22,0 & 25,7 & \multirow{2}{*}{0,000} & 58,4 & 40,2 & 49,4 & \multirow{2}{*}{0,000} \\
\hline Outras respostas & 68,4 & 78,0 & 74,3 & & 41,6 & 59,8 & 50,6 & \\
\hline $\mathrm{N}^{\circ}$ médio métodos que conhece & 9,6 & 9,3 & 9,4 & 0,892 & 11,4 & 11,1 & 11,2 & 0,568 \\
\hline Usou método na $1^{\mathrm{a}}$ relação & Branca & Negra & Total & Teste Qui ${ }^{2}$ & Branca & Negra & Total & Teste Qui ${ }^{2}$ \\
\hline Sim & 49,0 & 39,1 & 42,8 & \multirow{2}{*}{0,000} & 72,4 & 61,5 & 66,7 & \multirow{2}{*}{0,000} \\
\hline Não & 51,0 & 60,9 & 57,2 & & 27,6 & 38,5 & 33,3 & \\
\hline Uso método atual & Branca & Negra & Total & Teste Qui ${ }^{2}$ & Branca & Negra & Total & Teste Qui ${ }^{2}$ \\
\hline Sim & 89,1 & 85,0 & 86,5 & \multirow{2}{*}{0,000} & 87,3 & 87,1 & 87,2 & \multirow{2}{*}{0,000} \\
\hline Não & 10,9 & 15,0 & 13,5 & & 12,7 & 12,9 & 12,8 & \\
\hline Tipo método atual & Branca & Negra & Total & Teste Qui ${ }^{2}$ & Branca & Negra & Total & Teste Qui ${ }^{2}$ \\
\hline Esterilização feminina & 43,4 & 49,5 & 47,2 & \multirow{5}{*}{0,000} & 24,0 & 29,2 & 26,5 & \multirow{5}{*}{0,000} \\
\hline Pílula & 30,0 & 27,1 & 28,2 & & 42,3 & 31,7 & 37,1 & \\
\hline Camisinha & 15,6 & 15,1 & 15,3 & & 21,3 & 24,2 & 22,7 & \\
\hline Outro método moderno* & 6,3 & 5,9 & 6,0 & & 7,2 & 11,4 & 9,3 & \\
\hline Método tradicional** & 4,7 & 2,5 & 3,4 & & 5,3 & 3,5 & 4,4 & \\
\hline $\begin{array}{l}\text { Consultou médico quando } \\
\text { começou a usar pílula }\end{array}$ & Branca & Negra & Total & Teste Qui ${ }^{2}$ & Branca & Negra & Total & Teste Qui ${ }^{2}$ \\
\hline Sim & 78,4 & 71,6 & 74,4 & \multirow{2}{*}{0,005} & 89,2 & 84,0 & 86,7 & \multirow{2}{*}{0,007} \\
\hline Não & 21,6 & 28,4 & 25,6 & & 10,8 & 16,0 & 13,3 & \\
\hline $\begin{array}{l}\text { Tem ou não demanda por } \\
\text { contracepção }\end{array}$ & Branca & Negra & Total & Teste Qui ${ }^{2}$ & Branca & Negra & Total & Teste Qui ${ }^{2}$ \\
\hline Não tem demanda & 45,8 & 43,9 & 44,4 & \multirow{3}{*}{0,857} & 59,0 & 54,7 & 56,6 & \multirow{3}{*}{0,008} \\
\hline Tem demanda para espaçar & 23,4 & 23,4 & 23,4 & & 28,6 & 23,4 & 25,7 & \\
\hline Tem demanda para limitar & 30,8 & 32,8 & 32,2 & & 12,5 & 21,9 & 17,7 & \\
\hline
\end{tabular}

Fonte: PNDS, 2006 
A tabela 4 mostra os modelos de regressão logística para os dois principais indicadores apresentados acima: uso de método contraceptivo atual e existência/tipo de demanda por contracepção. Observa-se que a raça/cor somente foi uma variável estatisticamente significativa para o uso de método. Em que as mulheres negras apresentam $61 \%$ mais chances de não utilizarem métodos contraceptivos quando comparadas às mulheres brancas. Resultados similares foram encontrados por Costa e Carvalho (2014), em que no estudo também as negras apresentavam menos chances de uso do que as brancas. Para a demanda por contracepção parece que outras variáveis individuais, tais como estrato econômico, idade e número de filhos nascidos vivos possuem maior poder explicativo. Carvalho (2018) também não encontrou efeito da raça para a demanda não atendida por contracepção no Brasil após o controle dos dados por outras características sociodemográficas.

\begin{tabular}{|c|c|c|c|c|c|c|}
\hline \multirow{2}{*}{$\begin{array}{c}\text { Variáveis } \\
\text { independentes }\end{array}$} & \multirow{2}{*}{ Categorias } & \multicolumn{2}{|c|}{$\begin{array}{l}\text { Modelo } 4 \text { - uso de método } \\
\text { contraceptivo }(\mathrm{N}=7947)\end{array}$} & \multicolumn{3}{|c|}{$\begin{array}{r}\text { Modelo } 5 \text { - demanda } \\
\text { por contracepção }(\mathrm{N}=1080)\end{array}$} \\
\hline & & OddsRatio & P-valor & $\begin{array}{l}\text { OddsRatio } \\
\text { Espaçar }\end{array}$ & $\begin{array}{l}\text { OddsRatio } \\
\text { Limitar }\end{array}$ & P-valor \\
\hline \multirow{2}{*}{ Raça/cor } & Branca & Referência & \multirow{2}{*}{0,002} & Refe & ência & \multirow{2}{*}{0,382} \\
\hline & Negras & 1,614 & &, 759 & 1,328 & \\
\hline \multirow{2}{*}{ Escolaridade } & Alta & Referência & \multirow{2}{*}{0,017} & Refe & ência & \multirow{2}{*}{0,082} \\
\hline & Baixa & 1,427 & & ,569 & 1,250 & \\
\hline \multirow{3}{*}{$\begin{array}{l}\text { Estratos } \\
\text { Econômicos }\end{array}$} & Estratos A e B & Referência & \multirow{3}{*}{0,000} & Refe & ência & \multirow{3}{*}{0,000} \\
\hline & Estrado C & 1,184 & & ,472 & 19,942 & \\
\hline & Estrado De E & 1,965 & & ,601 & 18,900 & \\
\hline \multirow{3}{*}{ Religião } & Católica & Referência & \multirow{3}{*}{0,028} & Refe & ência & \multirow{3}{*}{0,057} \\
\hline & Evangélica & 1,078 & &, 851 & 1,757 & \\
\hline & Outras & 1,682 & & 1,970 & 1,757 & \\
\hline \multirow{5}{*}{$\begin{array}{l}\text { Macro região } \\
\text { administrativa }\end{array}$} & Sudeste & Referência & \multirow{5}{*}{0,001} & Refe & ência & \multirow{5}{*}{0,535} \\
\hline & Norte & 1,043 & & 1,080 & 1,333 & \\
\hline & Nordeste & ,641 & & 1,293 & 1,048 & \\
\hline & Sul & ,953 & & ,504 & 1,068 & \\
\hline & Centro-Oeste &, 783 & & ,799 & 1,129 & \\
\hline \multirow{2}{*}{$\begin{array}{l}\text { Local de } \\
\text { residência }\end{array}$} & \multirow{2}{*}{$\begin{array}{c}\text { Urbano } \\
\text { Rural }\end{array}$} & \multirow{2}{*}{$\begin{array}{l}\text { Referência } \\
, 892 \\
\end{array}$} & \multirow{2}{*}{0,601} & \multicolumn{2}{|c|}{ Referência } & \multirow{2}{*}{0,456} \\
\hline & & & & ,758 & ,673 & \\
\hline $\begin{array}{c}\mathrm{N}^{\circ} \text { filhos } \\
\text { nascidos vivos }\end{array}$ & Contínua & ,262 & 0,000 & 2,693 & 5,667 & 0,000 \\
\hline $\begin{array}{c}\text { Idade da mulher } \\
\text { em anos }\end{array}$ & Contínua & 1,052 & 0,000 & ,873 & 1,030 & 0,000 \\
\hline
\end{tabular}

Fonte: PNDS, 2006

Por fim, quanto ao acesso à saúde materna e assistência obstétrica as informações da Tabela 5 revelam que, apesar da maioria das mulheres, independentemente da raça/cor, terem feito a primeira consulta antes dos 5 meses, o percentual daquelas que a fizeram depois de 5 meses e é ligeiramente maior para mulheres negras. A média de meses é muito próxima entre os grupos analisados. Já os dados de proteção mínima de assistência pré-natal (seis consultas ao 
longo da gestação, mais uma consulta no puerpério), foi bastante distinto entre os grupos de escolaridade, e não tão diferente entre os grupos raciais. O número médio de consultas pré-natal supera o número recomendado de consultas e foi de 7,4 para as mulheres de baixa escolaridade e de 8,8 entre as mais escolarizadas. É importante notar que entre o percentual daquelas mulheres que fazem o número de consultas recomendas é mais elevado entre as negras do que as brancas.

\begin{tabular}{|c|c|c|c|c|c|c|c|c|}
\hline \multirow{2}{*}{$\begin{array}{c}\text { Indicadores } \\
\text { Quantos meses tinha quando fez a } \\
\text { primeira consulta de pré-natal }\end{array}$} & \multicolumn{4}{|c|}{ Baixa } & \multicolumn{4}{|c|}{ Alta } \\
\hline & Branca & Negra & Total & $\begin{array}{l}\text { Test } \\
\text { Qui }^{2}\end{array}$ & Branca & Negra & Total & $\begin{array}{l}\text { Test } \\
\text { Qui }^{2}\end{array}$ \\
\hline Até 4 meses & 94,6 & 92,5 & 93,2 & \multirow{2}{*}{0,056} & 98,6 & 94,9 & 96,4 & \multirow{2}{*}{0,021} \\
\hline 5 meses ou mais & 5,4 & 7,5 & 6,8 & & 1,4 & 5,1 & 3,6 & \\
\hline $\begin{array}{l}\text { Média de meses na } 1^{\mathrm{a}} \text { consulta } \\
\text { pré-natal }\end{array}$ & 2,3 & 2,6 & 2,5 & & 1,64 & 2,01 & 1,8 & \\
\hline $\begin{array}{l}\text { Quantas consultas de pré-natal fez } \\
\text { durante a gravidez }\end{array}$ & Branca & Negra & Total & $\begin{array}{l}\text { Test } \\
\text { Qui }^{2} \\
\end{array}$ & Branca & Negra & Total & $\begin{array}{l}\text { Test } \\
\text { Qui }^{2} \\
\end{array}$ \\
\hline Menos que 3 consultas & 1,6 & 2,4 & 2,1 & \multirow{3}{*}{0,083} & 0,2 & 0,4 & 0,3 & \multirow{3}{*}{0,000} \\
\hline De 3 a 5 consultas & 16,9 & 18,3 & 17,8 & & 5,3 & 10,4 & 8,3 & \\
\hline 6 consultas ou mais & 81,5 & 79,3 & 80,1 & & 94,5 & 89,2 & 91,4 & \\
\hline $\begin{array}{l}\text { Número médio de consultas pré- } \\
\text { natais }\end{array}$ & 7,71 & 7,12 & 7,4 & 0,589 & 9,24 & 8,37 & 8,8 & 0,589 \\
\hline Local onde foi feito o parto & Branca & Negra & Total & $\begin{array}{l}\text { Test } \\
\text { Qui }^{2}\end{array}$ & Branca & Negra & Total & $\begin{array}{l}\text { Test } \\
\text { Qui }^{2}\end{array}$ \\
\hline Domicílio & 0,6 & 1,9 & 1,4 & \multirow{3}{*}{0,000} & 0,0 & 0,4 & 0,2 & \multirow{3}{*}{0,000} \\
\hline Público & 82,9 & 87,8 & 86,1 & & 45,7 & 65,7 & 57,3 & \\
\hline Particular & 16,5 & 10,3 & 12,5 & & 54,3 & 33,9 & 42,5 & \\
\hline $\begin{array}{l}\text { Algum procedimento realizado } \mathrm{p} / \\
\text { aliviar a dor no parto }\end{array}$ & Branca & Negra & Total & $\begin{array}{l}\text { Test } \\
\text { Qui }^{2}\end{array}$ & Branca & Negra & Total & $\begin{array}{l}\text { Test } \\
\text { Qui }^{2}\end{array}$ \\
\hline Sim & 31,3 & 25,1 & 27,2 & \multirow{2}{*}{0,146} & 39,2 & 25,6 & 30,6 & \multirow{2}{*}{0,634} \\
\hline Não & 61,9 & 65,4 & 64,2 & & 55,4 & 65,1 & 61,5 & \\
\hline $\begin{array}{l}\text { Companheiro/família/amigo ficou } \\
\text { junto durante o parto }\end{array}$ & Branca & Negra & Total & $\begin{array}{l}\text { Test } \\
\text { Qui }^{2}\end{array}$ & Branca & Negra & Total & $\begin{array}{l}\text { Test } \\
\text { Qui }^{2}\end{array}$ \\
\hline Sim & 13,3 & 12,7 & 12,9 & \multirow{2}{*}{0,000} & 29,9 & 18,0 & 23,0 & \multirow{2}{*}{0,000} \\
\hline Não & 86,7 & 87,0 & 86,9 & & 70,1 & 82,0 & 77,0 & \\
\hline Fez exame pós-parto & Branca & Negra & Total & $\begin{array}{l}\text { Test } \\
\text { Qui }^{2} \\
\end{array}$ & Branca & Negra & Total & $\begin{array}{l}\text { Test } \\
\text { Qui }^{2}\end{array}$ \\
\hline Sim & 37,1 & 27,9 & 31,1 & \multirow{2}{*}{0,000} & 56,1 & 47,1 & 50,8 & \multirow{2}{*}{0,001} \\
\hline Não & 62,9 & 72,1 & 68,9 & & 43,9 & 52,9 & 49,2 & \\
\hline $\begin{array}{l}\text { Realizou todos os procedimentos } \\
\text { básicos }\end{array}$ & 57,9 & 68,4 & 64,8 & 0,000 & 66,4 & 70,7 & 68,8 & 0,055 \\
\hline
\end{tabular}

Fonte: PNDS, 2006

Sobre as características relativas ao parto, o local do parto, a maioria deles foi feito em hospitais públicos, especialmente para as negras. As mulheres brancas de alta escolaridade mais da metade delas utilizavam a rede privada de hospitais para ter seus filhos, enquanto as negras deste mesmo grupo apenas $34 \%$ o faziam. A maior parte das mulheres não receberam medicamento para alívio do parto vaginal, com nenhuma diferença entre raça/cor. Para ambos os grupos de mulheres, um pequeno percentual teve o direito de ter acompanhante/amigo durante o 
parto, em que foi ainda menos acessível para as mulheres negras. Quanto à realização do exame de pós-parto, apesar de pequenas as diferenças, novamente as negras estão sobre representadas entre aquelas que não fazem o exame para ambos os grupos educacionais.

De modo geral, pode-se dizer que houve grandes avanços nestes indicadores mostrando melhorias no acesso à saúde reprodutiva quando comparados os dados de 1996 analisados por Perpétuo (2000), especialmente aquelas relativos ao pré-natal. Contudo, para alguns indicadores, ainda persistem diferenças em que a raça/cor parece ser um fator importante. Resultados similares sobre o local do parto também foram encontrados por Mandarino et al. (2009). Leal et al. (2005) e Silva et al. (2011) indicam que, mesmo após o controle estatístico de outras variáveis de confundimento, as mulheres negras continuam em desvantagem no acesso ao pré-natal e medicamento para alivio da dor. Berquó e Lago (2016), mostraram que na assistência à gestação, inicialmente, havia diferenças desfavorecendo as mulheres negras, no que se refere à realização de no mínimo 6 consultas no pré-natal, de parto cesáreo, parto com presença de acompanhante e realização de consulta no puerpério, contudo estas diferenças não foram estatisticamente significativas quando se analisou por estratos socioeconômicos.

\section{Discussão e Considerações Finais}

Pode-se concluir que com relação à experiência contraceptiva e reprodutiva das mulheres, as negras continuam iniciando a vida sexual e tendo o primeiro filho mais cedo do que as brancas e, muitas, ainda na adolescência. Ao comparar com dados da década de 90 (PERPÉTUO, 2000) concluímos que esse quadro pouco se alterou nos últimos dez anos. Contudo, quando se analisa por escolaridade, pode-se concluir que estes são ainda mais acirrados do que entre os grupos raciais. Exceto para o indicador de parturição ( $n^{\circ}$ médio de filhos nascidos vivos), em que há tanto diferenças por escolaridade (a maior) quanto por raça/cor, parece que o efeito da escolaridade é maior do que os diferenciais por raça/cor.

Percebeu-se uma grande mudança nos indicadores relativos à experiência contraceptiva, em que atualmente existem menores diferenças por raça/cor no uso de contracepção, uma vez que um percentual importante de mulheres utilizava método contraceptivo independentemente do grupo racial. Porém é importante destacar que poucas mulheres negras de baixa escolaridade utilizavam método na primeira relação, e assim como foi já demonstrado para a década de 90, as mulheres negras continuavam sendo a maioria entre aquelas esterilizadas e também apresentavam um percentual ligeiramente maior entre as que não utilizavam nenhum método na data da pesquisa, independentemente da escolaridade. Além disso, grande parte das mulheres mais escolarizadas obteve o método contraceptivo em meios particulares, principalmente as 
mulheres brancas; enquanto uma parcela considerável das mulheres menos escolarizadas, sobretudo as negras, obtiveram seus métodos através do SUS. Estes diferenciais levam a desigualdades importantes na demanda insatisfeita por planejamento da fecundidade, em que os resultados mostraram que as negras, independentemente do nível de escolaridade, têm maior dificuldade em implementarem suas preferências reprodutivas.

Sobre os indicadores de saúde materna, conclui-seque houve melhoria significativa em relação ao ano de 1996, com permanência das diferenças por raça/cor encontrados anteriormente. Contudo, quando se controla por escolaridade, observa-se que negras quanto brancas realizaram em média a quantidade de consultas de pré-natal aconselhada e também realizaram consultas logo no início da gravidez. As negras também apresentaram bons indicadores em relação aos procedimentos básicos, grande parte delas realizou todos os exames. De tal modo, os resultados indicam que no acesso à saúde materna os diferenciais estão mais relacionados às diferenças nas condições de vida das mulheres do que a sua raça/cor.

Apesar do acesso das mulheres negras à saúde sexual e reprodutiva ter apresentado avanços, uma vez que a maior parte delas já utilizam algum método para planejar sua fecundidade e em alguns outros indicadores, os diferenciais estarem mais relacionados à diferenças de escolaridade, o estudo mostrou que ainda hoje há influência da raça/cor no acesso de muitas mulheres ao direito à uma vida sexual e reprodutiva segura. Os resultados encontrados mostraram que, de maneira geral, os indicadores das brancas de alta escolaridade eram sempre mais satisfatórios, seguidos pelas negras de alta escolaridade, brancas de baixa escolaridade e, por fim, negras de baixa escolaridade.

Além disso, é importante ressaltar que, por indisponibilidade dos dados na pesquisa utilizada, sabe-se que esses diferenciais observados podem ser resultados para além das desigualdades sociais aqui colocadas. Ou seja, podem ser consequências de outros fatores que não são captados pelas pesquisas quantitativas, como, por exemplo, a forma diferenciada de tratamento e atendimento dado às mulheres negras simplesmente pelo fato de serem negras, às dificuldades de informação e acesso devido à raça/cor, dentre outros aspectos vinculados à discriminação racial e racismo direto sofrido pelo grupo das negras. O que, para além das desigualdades sociais, contribuiriam para os piores resultados no acesso à saúde sexual e reprodutiva desse grupo racial.

\section{Referências Bibliográficas}

AERTS, D. R. G. C. et al. Fatores associados ao início da vida sexual ativa de escolares em uma cidade do sul do Brasil. Aletheia, v.45, p.87-100, set./dez. 2014. 
BARROS, J. V. de S.; WONG, L. R. Medindo a saúde reprodutiva segundo o tipo de união na América Latina: indicadores sintéticos para Brasil e México. 2013. xii, $101 \mathrm{f}$.

BERQUÓ, E. Esterilização e raça em São Paulo. Revista Brasileira de Estudos Populacionais, Campinas, v. 11, n. 1, p. 19-26, jan./jun. 1994.

BERQUÓ, E; GARCIA, S; LIMA, L Reprodução na juventude: perfis sociodemográficos, comportamentais e reprodutivos na PNDS 2006. Revista de Saúde Pública, São Paulo, v. 46, n. 4, 10 jul. 2012.

BERQUÓ, E; LAGO, T. D. G. Atenção em saúde reprodutiva no Brasil: eventuais diferenciais étnico-raciais. Saúde Sociedade. São Paulo, v.25, n.3, p.550-560, 2016.

CARVALHO, A. A. Demanda por contracepção no brasil em 2006: Contribuição para a implementação das preferências de fecundidade. Ciência e Saúde Coletiva [periódico na internet] (2018/Abr).

CAVAS, C. S. T.; D'ÁVILA NETO, M. I. Diáspora Negra: Desigualdades de gênero e raça no Brasil. In: Fazendo Gênero, 9., 2010, Santa Catarina. Anais... Santa Catarina: UFSC, 2010, p. 19.

COUTINHO, R. Z; GOLGHER, A. B. Modelando os determinantes próximos da fecundidade para o Brasil: o advento das preferências competitivas. Revista Brasileira de Estudos de População, v. 35, n. 1, p. 1-28, 2018.

CORRÊA, S; ALVES, J. E. D.; JANNUZZI, P. de M. Direitos e saúde sexual e reprodutiva: marco teórico-conceitual e sistema de indicadores. In: CAVENAGHI, S. Indicadores municipais de saúde sexual e reprodutiva. Rio de Janeiro: ABEP, Brasília: UNFPA, 2006. cap.1, p. 27-62.

COSTA. I. G.D.; CARVALHO, A. A.Uso de contracepção por mulheres de diferentes grupos religiosos: diferenças ou semelhanças? Horizonte, v. 12, n. 36, p. 1114-1139, out./dez. 2014

DE SOUSAI, B. C. et al. Comportamento sexual e fatores associados em adolescentes da zona rural. Revista de Saude Publica, v. 52, p. 39, 2018.

FRANCISCO, P. M. S. B.; DONALISIO, M. R.; BARROS, M. B. A.; CESAR, C. L.G.; CARANDINA, L.; GOLDBAUM, M. Medidas de associação em estudo transversal com delineamento complexo: razão de chances e razão de prevalência. Revista Brasisleira de Epidemiologia, v. 11, n.3, p. 347-55, 2008.

GUEDES, G. G.; GUEDES, T. A. O etnocentrismo e a construção racismo. Periódico Científico Outras Palavras, v. 10, n. 1, jun. 2014.

HASENBALG, C.; SILVA, N. do V. (eds.). (1988),Estrutura Social, Mobilidade e Raça. Rio de Janeiro, Iuperj/Vértice.

IBGE. Pesquisa Nacional por Amostra de Domicílios Contínua : características gerais dos moradores : 2012-2016. Rio de Janeiro, 2017 Disponível em: https://biblioteca.ibge.gov.br/index.php/biblioteca-catalogo?view=detalhes\&id=2101377. Acesso em 20/01/2018.

LACERDA, M. A. Perfis de demanda insatisfeita por contracepção nos municípios de Belo Horizonte e Recife, 2002. 141 f. Dissertação (Mestrado em Demografia) - Cedeplar/ UFMG, 2005.

LEAL M.C.; GAMA, S.G.N.; CUNHA, C. B. Desigualdades raciais, sociodemográficas e na assistência ao pré-natal e ao parto, 1999-2001. Revista de Saúde Pública, v.39, p. 100-7, 2005. 
LESSA I.; MAGALHÃES L.; ARAÚJO MJ, ALMEIRA FILHO N, AQUINO E, OLIVEIRA MMC. Hipertensão arterial na população adulta de Salvador (BA) - Brasil. ArqBrasCardio, v.87, p.747-56, 2006.

LOPES, F. Para além da barreira dos números: desigualdades raciais e saúde. Cadernos de Saúde Pública, Rio de Janeiro, v. 21, n. 5, p. 1595-1601, set./out. 2005.

MADISON, T.; SCHOTTENFELD, D.; JAMES, S. A.; SCHWARTZ, A. G.; GRUBER, S.B. Endometrial cancer: Socioeconomic status and racial/ethnicdifferences in stageatdiagnosis, treatment, andsurvival. Am J PublicHealt, v.94, p.2104-11, 2004.

MANDARINO, N. R. et al. Aspectos relacionados à escolha do tipo de parto: um estudo comparativo entre uma maternidade pública e outra privada, em São Luís, Maranhão, Brasil. Cadernos de Saúde Pública, Rio de Janeiro, 2009.

MARTINS, A. L. Mortalidade materna de mulheres negras no Brasil. Cadernos de Saúde Pública, v. 22, n. 11, p. 2473-2479, nov. 2006.

MESENBURG, M. A. et al.Ethnicgroupinequalities in coveragewithreproductive, maternal andchildhealthinterventions: cross-sectionalanalysesofnationalsurveys in 16 Latin American andCaribbean countries. The Lancet Global Health, v. 6, n. 8, p. 902-913, 2018.

MINISTÉRIO DA SAÚDE, Secretaria de Atenção à Saúde, Departamento de Ações Programáticas Estratégicas. Pré-natal e Puerpério: Atenção Qualificada e Humanizada: manual técnico. Brasília: Ministério da Saúde, 2006. 162p.

PESQUISA NACIONAL DE DEMOGRAFIA E SAÚDE DA CRIANÇA E DA MULHER. Relatório. Brasília, 2008. 306 p.

MONTEIRO, S.; MAIO, M. C. Etnicidade, raça e saúde. No brasil: questões e desafios. In: MONTEIRO, S. e SANSONE, L. (Org.) Etnicidade na América Latina: um debate sobre raça, saúde e direitos reprodutivos. Rio de Janeiro: Fundação Oswaldo Cruz; 2004.

MONTEIRO, S.; SANSONE, L. Etnicidade na America Latina: um debate sobre raça, saúde e direitos reprodutivos. Rio de Janeiro: FIOCRUZ, 2004.

OLINTO, M. T. A.; OLINTO, B. A. Raça e desigualdade entre as mulheres: um exemplo no sul do Brasil. Cadernos de Saúde Pública, v.16, n.4, p. 1137-1142, 2000.

OLIVEIRA, F. O Recorte Racial/Étnico e a Saúde Reprodutiva: mulheres negras. Questões da Saúde Reprodutiva. Editora FIOCRUZ, p. 419-438, 1999.

PERPÉTUO, I. H. O. Raça e acesso às ações prioritárias na agenda da saúde reprodutiva. Encontro Nacional de Estudos Populacionais, 12, 2000, Caxambú. Anais... Belo Horizonte: ABEP, 2000

SANTOS, S. M. dos; GUIMARAES, M. J. B.; ARAUJO, T. V. B. de.Desigualdades raciais na mortalidade de mulheres adultas no Recife, 2001 a 2003. Saúde Coletiva,vol.16, n.2, pp. 87$102,2007$.

SANTOS, S. Saúde. Condições De Vida Das Mulheres Negras Em Pernambuco. Recife, Sos Corpo - Instituto Feminista para a Democracia, 2015. Disponível em: http://soscorpo.org/wpcontent/uploads/Condicoes-de-Vida-das-Mulheres-Negras-em-Pernambuco.pdf. Acesso em: 01 fev. 2018

SIMÃO, A. B; MIRANDA-RIBEIRO, P.; CAETANO, A. J. O recorte raça/cor e a saúde reprodutiva em Belo Horizonte e Recife: uma análise exploratória sobre a realização de consultas ginecológicas, 2002. Encontro Nacional de Estudos Populacionais, 14, 2004, Caxambu. Anais... Belo Horizonte: ABEP, 2002. 
SILVA, Z. P. et al. Perfil sociodemográfico e padrão de utilização dos serviços de saúde do Sistema Único de Saúde (SUS), 2003-2008. Ciência e Saúde Coletiva, Rio de Janeiro, v. 16, n. 9, p. 3807-3816, 2011.

WESTOFF, C. F.; OCHOA, L. H. Unmetneedandthedemand for familyplanning. Demographic and Health Surveys Comparatives Studies. Columbia (USA) Maryland: Institute for Resource Development/Macro Systems, Inc., n. 5. 1991.

Agência Financiadora: Instituto Brasileiro de Geografia e Estatística (IBGE)

Recebido em 27/05/2020 - Aprovado em 25/08/2020. 